\title{
A comparative study of saline infusion sonohysterography and hysterosalpingography for evaluation of female infertility
}

\author{
Riya Bhattacharya*, A. C. Ramesh
}

Department of Obstetrics and Gynecology, JJM Medical College, Davangere, Karnataka, India

Received: 13 October 2019

Revised: 03 December 2019

Accepted: 11 December 2019

\author{
*Correspondence: \\ Dr. Riya Bhattacharya, \\ E-mail: destiny4riya@gmail.com
}

Copyright: () the author(s), publisher and licensee Medip Academy. This is an open-access article distributed under the terms of the Creative Commons Attribution Non-Commercial License, which permits unrestricted non-commercial use, distribution, and reproduction in any medium, provided the original work is properly cited.

\begin{abstract}
Background: Infertility is a complex disorder with significant psychological and emotional impact. It affects $10-15 \%$ of couples in the reproductive age group. Hence, evaluation of female genital tract is an important part of workup of an infertile woman. The most commonly used methods are hysterosalpingography which exposes the patient to ionizing radiation and laparoscopy which is invasive. This calls for the need of a low risk method that would be suited for ambulatory application. Saline infusion sonohysterography is a cost-effective, safe, non-invasive and a rapid procedure to visualize the female pelvic organ in evaluation of infertility. The objective of this study was to compare diagnostic accuracy of saline infusion sonography (SIS) over conventional hysterosalingography (HSG) for evaluation of female infertility.

Methods: Total 50 patients who presented to the gynecology OPD between 2018 to September 2019 for evaluation of infertility were included for the study. The results of the two procedures were compared.

Results: For evaluation of uterine cavity, SIS had a sensitivity of 95\%, specificity of 100\%, PPV of $100 \%$, NPV $75 \%$ and a sensitivity of $100 \%$, specificity of $100 \%$, PPV of $91 \%$, NPV of $100 \%$ for tubal patency as compared to HSG.

Conclusions: For evaluation of uterine cavity, SIS had a sensitivity of $95 \%$, specificity of $100 \%$, PPV of $100 \%$, NPV $75 \%$ and a sensitivity of $100 \%$, specificity of $100 \%$, PPV of $91 \%$, NPV of $100 \%$ for tubal patency as compared to HSG.
\end{abstract}

Keywords: Hysterosalpingography, Infertility, Saline infusion sonohysterography

\section{INTRODUCTION}

Infertility is defined as the inability of a couple to conceive after 1 year of coital activity without any contraception. $^{1}$ Around $10 \%$ of women are facing problems of infertility globally. ${ }^{2}$ The etiology of infertility is multi factorial, uterine and tubal factors accounting for $10 \%$ and $25-30 \%$ respectively for infertility. Hence, evaluation of female genital tract is of utmost importance. The most commonly used methods for this purpose are laparoscopy, which is invasive, and HSG which exposes the patient to ionizing radiation. ${ }^{3}$
Patients fear of anaesthesia, surgery or radiation exposure and the objective risks of intervention often delay the testing of tubal patency and this approach may lead to long periods of expensive attempts at treatment with ovulation inducing drugs before the patient is finally referred to a hospital for tubal diagnosis. ${ }^{3}$ This calls for a low risk method that would be suited for ambulatory application. In this study authors have attempted to evaluate the utility of SIS. HSG is a procedure which exposes the patient to ionizing radiation and contrast medium. It is routinely practised in the workup of an infertile patient and provides information about uterine 
cavity - its size, shape contour, filling defects suggestive of polyp, septa, adhesions, fibroids and tubal patency. Laparoscopy with chromopertubation is considered as the gold standard for diagnosing tubal occlusion but is usually preferred for the assessment or treatment of other associated pelvic pathology. It is an invasive procedure also needing anaesthesia, thus adding to the cost and side effects. To alleviate the problem, an outpatient procedure has been thought out known as saline infusion sonohysterograhy (SIS) or sonosalpingography or hysterosonography. SIS is gaining popularity and is being widely practiced and accepted as a screening tool in assessing tubal patency and in evaluation of uterine cavity.

Objective of this study was to compare the diagnostic accuracy of saline infusion sonohysterography over conventional hysterosalpingography in the evaluation of female infertility.

\section{METHODS}

In a tertiary care hospital JJM Medical College, Davangere. An observational study was conducted from 1st October 2018 to 30th September 2019. A total 50 patients in the reproductive age group who were anxious to conceive and fulfilled the inclusion criteria.

\section{Inclusion criteria}

- Primary and secondary Infertility patients in reproductive age group.

\section{Exclusion criteria}

- Acute pelvic infection

- Acute vaginal bleeding

- Pregnancy

- Malignancy of the genital tract

- Abnormal semen analysis of the patient.

All eligible patients were counselled for both the procedures and informed written consent was taken. Detailed menstrual, obstetric, and medical history of each patient were obtained followed by general, physical, systemic, and gynaecological examination. The patient was given Inj. atropine intra-muscular $0.6 \mathrm{mg}$, Inj. tetanus toxoid $0.5 \mathrm{mg}$ and a broad spectrum intravenous antibiotic half an hour prior to the procedure.

\section{Procedure of saline infusion sonohysterography}

Under aseptic precautions, a baseline transvaginal ultrasound was performed with an empty bladder with 7.5 $\mathrm{MHz}$ transvaginal transducer. The patient was put in dorsal position, cervix and vagina was cleaned with a sterile swab. The cervix was visualized with the help of a sim's speculum and an anterior wall retractor. The uterine sound was passed, both to know the position and the size of the uterus as well as to rule out cervical stenosis. Then,
Foley's No. 8 catheter was directed into the uterine cavity and the balloon was inflated with $2 \mathrm{ml}$ of normal saline and pulled back such that it lies at the level of internal os. $10-40 \mathrm{ml}$ of normal saline was instilled trans cervically through the catheter to provide enhanced endometrial visualization during transvaginal ultrasound examination. Maximum of $50 \mathrm{ml}$ saline was instilled. ${ }^{4}$ The saline served as a negative contrast agent and once adequate distension was achieved the size, shape, and uterine and cervical echotexture in sagittal and transverse planes was measured. Endometrial regularity, thickness and echogenicity relative to myometrium was noted. The presence of any structural abnormality of endometrium was ruled out. ${ }^{5}$ The pouch of Douglas was visualized. Flow of fluid and air "turbulence" was looked for in the region of right and left ovarian fossae and this turbulence also known as "the waterfall sign" was taken as patency of the respective fallopian tube. ${ }^{4}$ Later, the cul-de-sac was also visualized to look for free fluid. In the presence of obstructed tube, the uterine cavity expanded in size and no waterfall sign was observed. This procedure was performed between $7^{\text {th }}$ and $12^{\text {th }}$ day of the menstrual cycle and the patients were kept for observation half an hour after the procedure.

\section{Procedure of hysterosalpingography}

HSG is a contrast enhanced fluoroscopic radiological procedure wherein radiographs were taken of a female reproductive tract after injection of a suitable contrast media. It was carried out between the $7^{\text {th }}$ and $12^{\text {th }}$ day of the menstrual cycle. The patient was placed in supine position on the radiographic table with the hips flexed. Under aseptic precautions, the cervix is visualised with the help of speculum and the anterior lip was held with a vulsellum. Next Rubin's cannula was inserted into the endocervical canal after sounding the uterus with a uterine sound. Water soluble contrast like Urografin $76 \%$ was used. $10 \mathrm{ml}$ of dye with $10 \mathrm{ml}$ of normal saline was filled in a $20 \mathrm{ml}$ syringe. Rubin's cannula was filled with the contrast material to flush out the air and then it was inserted into the external os under direct vision with counter traction applied on the vulsellum to ensure a tight seal. With fluoroscopic control, 15-20 ml of contrast was pushed into the uterine cavity. Spot radiographs during the phases of early uterine filling, tubal filling and 5 minutes later to detect the peritoneal spill were taken. A release film was taken to check for the clearance of contrast from the pelvic cavity. ${ }^{6}$ Contrast material was injected slowly to avoid any discomfort, spasm of the utero-tubal junction and to avoid non-visualization of lesions due to large quantity of contrast material. Once the radiographs were found to be satisfactory, the cannula was removed and the vagina cleaned, and the patient was kept under observation for half an hour.

\section{Statistical analysis}

Statistical analysis was done by using SPSS software version 16.0 


\section{RESULTS}

The present study was carried out in a total of 50 patients.

Table 1: Age of the patients.

\begin{tabular}{|lll|}
\hline Age & Total no. of cases & $\%$ \\
\hline 20-25 years & 8 & $16 \%$ \\
\hline 26-30 years & 16 & $32 \%$ \\
\hline 31-35 years & 16 & $32 \%$ \\
\hline 35-40 years & 8 & $16 \%$ \\
\hline$>$ 40 years & 2 & $4 \%$ \\
\hline
\end{tabular}

Table 1 shows that approximately $80 \%$ of the patients belonged to the reproductive age group of 20-35 years. The age group of 25-30 years and 30-35 year comprised maximum patients i.e. $32 \%$ in both. As the fertility of women decreases progressively with age particularly after 35 years, the earlier the patient is investigated, the better the chance of success.

Table 2: Type of infertility.

\begin{tabular}{|lll|}
\hline & No. of cases & $\%$ \\
\hline Primary infertility & 41 & $82 \%$ \\
\hline Secondary infertility & 9 & $18 \%$ \\
\hline
\end{tabular}

Table 2 shows that there were more cases of primary infertility, i.e. $82 \%$ and $18 \%$ of secondary infertility.

Table 3: Duration of infertility.

\begin{tabular}{|lll|}
\hline Years & No. of cases & $\%$ \\
\hline $1-5$ years & 14 & $28 \%$ \\
\hline $5-10$ years & 20 & $40 \%$ \\
\hline $10-15$ years & 12 & $24 \%$ \\
\hline$>15$ years & 4 & $8 \%$ \\
\hline
\end{tabular}

Table 3 depicts that maximum number of patients had 510 years infertility period i.e. $40 \%$ followed by $1-5$ years infertility. The shortest duration was 2 years.

Table 4: Evaluation of intra-uterine pathologies.

\begin{tabular}{|lll|}
\hline & SHG $(\mathbf{N}=\mathbf{4 7})$ & HSG $(\mathbf{N}=\mathbf{4 7})$ \\
\hline Normal & 39 & 41 \\
\hline Abnormalities & 8 & 6 \\
\hline Total & $\mathbf{4 7}$ & $\mathbf{3 5}$ \\
\hline
\end{tabular}

In the above (Table 4) 47 cases were evaluated for intrauterine pathologies. Out of which saline infusion sonohysterogrphy picked up 8 of the abnormalities as compared to 6 by hysterosalpingography.

In the above study, three patients were not included in the study a; one due to tight stenosis of cervical canal on insertion of catheter during SIS and 2 patients were excluded due to non- compliance pertaining to giddiness, nausea and palpitations.

Table 5: Various uterine cavity abnormalities.

\begin{tabular}{|lll|}
\hline \multicolumn{1}{|c|}{} & $\begin{array}{l}\text { SHG } \\
(\mathbf{N}=47)\end{array}$ & $\begin{array}{l}\text { HSG } \\
(\mathbf{N}=47)\end{array}$ \\
\hline Endo metrialpolyp & 2 & 2 \\
\hline Submucous fibroid & 2 & 1 \\
\hline Septate uterus & 1 & 1 \\
\hline Intra-uterine adhesion & 1 & 1 \\
\hline Other fibroids & 2 & 1 \\
\hline Total & $\mathbf{8}$ & $\mathbf{6}$ \\
\hline
\end{tabular}

Table 6: Tubal pathologies on SIS and HSG.

\begin{tabular}{|lll|}
\hline & SHG & HSG \\
\hline Tubo-ovarian mass & $7(14.8 \%)$ & $5(10.6 \%)$ \\
\hline Hydro-salpinx & $4(8.5 \%)$ & $3(6.3 \%)$ \\
\hline
\end{tabular}

Table 5 highlights the detection uterine abnormalities by both HSG and SIS. SIS diagnosed $8.51 \%$ of cases as fibroids, whereas HSG detected only $4.25 \%$ of cases. HSG missed one submucous fibroid and intra-mural fibroid which was detected on SIS. This shows that SIS is more sensitive and superior as compared to HSGin diagnosing uterine pathology.

In Table $6,14.8 \%$ of the cases had tubo-ovarian masses detected by SIS as compared to $10.6 \%$ by HSG. Hydrosalpinx was found in $8.5 \%$ of subjects by SIS and in $6.3 \%$ by $\mathrm{HSG}$.

Table 7: Tubal patency on SIS and HSG.

\begin{tabular}{|lll|}
\hline & SHG & HSG \\
\hline B/L tubal patency & 35 & 33 \\
\hline B/L tubal blockage & 4 & 6 \\
\hline U/L tubal blockage & 8 & 8 \\
\hline
\end{tabular}

In Table 7, tubal patency was assessed first by SIS, which was later compared with HSG, which was considered the gold standard. About $74.4 \%$ had bilateral tubal patency in SIS.

Whereas, $70.21 \%$ showed bilateral patency in HSG. Bilateral tubal occlusion was seen in $8.51 \%$ in SIS, whereas it was $12.7 \%$ in HSG. Unilateral patency of $17.02 \%$ was seen in SIS, and in HSG.

Table 8 summarizes the findings of the current study showing a sensitivity of $95 \%$, specificity of $100 \%$, PPV of $100 \%$, NPV $75 \%$ of SIS for evaluation of uterine cavity. For tubal patency, when compared to HSG, SIS had a sensitivity of $100 \%$, specificity of $100 \%$, PPV of $91 \%$, NPV of $94 \%$. SIS is found to be more sensitive and specific to conventional HSG in the evaluation of uterine and tubal factors of female infertility. 
Table 8: Uterine pathology.

\begin{tabular}{|llll|}
\hline $\begin{array}{l}\text { Saline infusion } \\
\text { sonohysterography } \\
\text { (SIS) }\end{array}$ & $\begin{array}{l}\text { Hysterosalpingography } \\
\text { (HSG) }\end{array}$ & Total \\
\hline Normal & Abnormal & \\
\hline Normal & 39 & 0 & 39 \\
\hline Abnormal & 2 & 6 & 8 \\
\hline Total & $\mathbf{4 1}$ & $\mathbf{6}$ & $\mathbf{4 7}$ \\
\hline Validity measures & & & SIS \\
\hline Sensitivity & & $95 \%$ \\
\hline Specificity & & $100 \%$ \\
\hline Positive predictive value & & $100 \%$ \\
\hline Negative predictive value & & $75 \%$ \\
\hline Diagnostic accuracy & & $96 \%$ \\
\hline
\end{tabular}

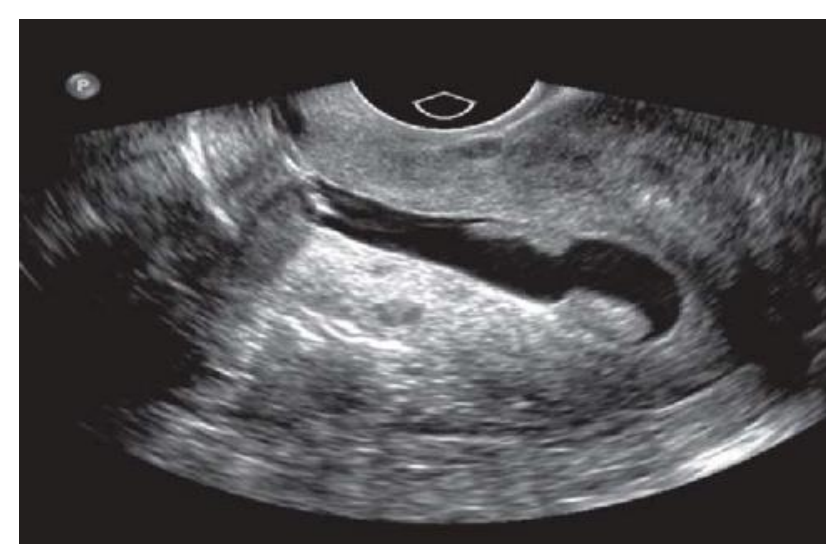

Figure 1: Endometrial polyp on saline infusion sonography.

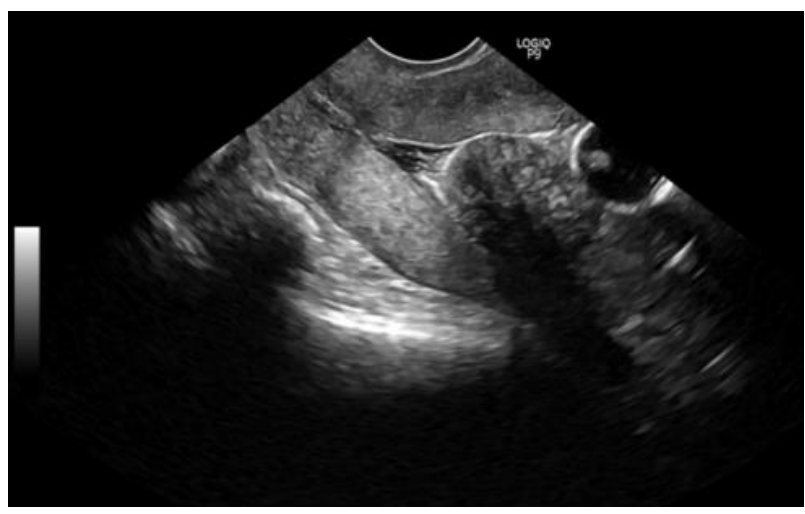

Figure 2: A submucous fibroid is delineated on saline infusion.

Figure 1 illustrates an endometrial polyp on saline infusion sonography. A submucous fibroid is delineated on saline infusion in Figure 2. Figure 3 outlines an anterior wall intramural fibroid on Saline infusion sonography. Figure 4 shows the bilateral spillage of dye on a normal HSG. Figure 5 visualizes a bilateral hydrosalpinx without any spillage of dye on HSG. Figure 6 represents a right sided distal tubal blockage with hydrosalpinx on HSG.

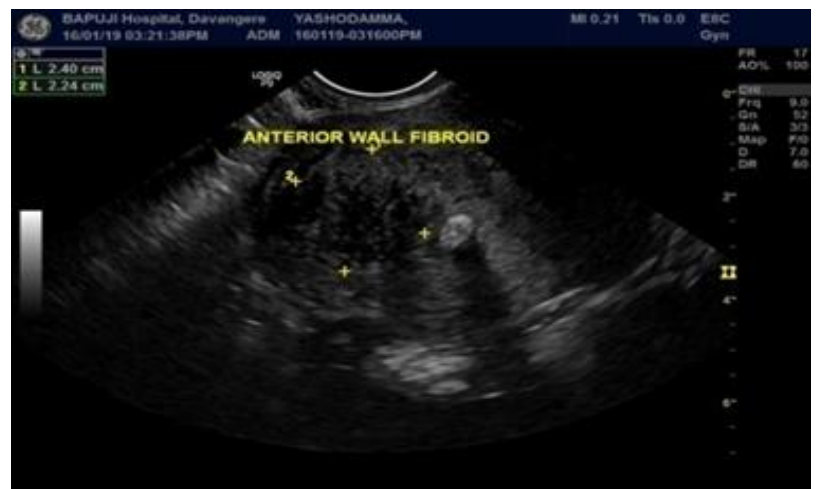

Figure 3: An anterior wall intramural fibroid on saline infusion sonography.

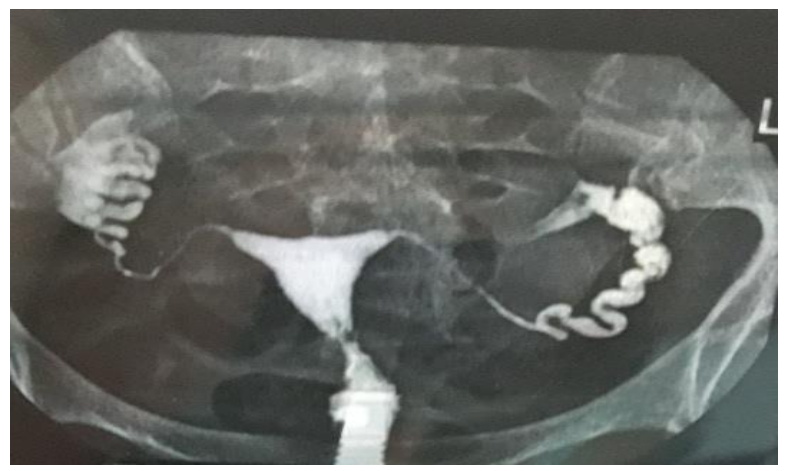

Figure 4: Bilateral spillage of dye on a normal HSG.

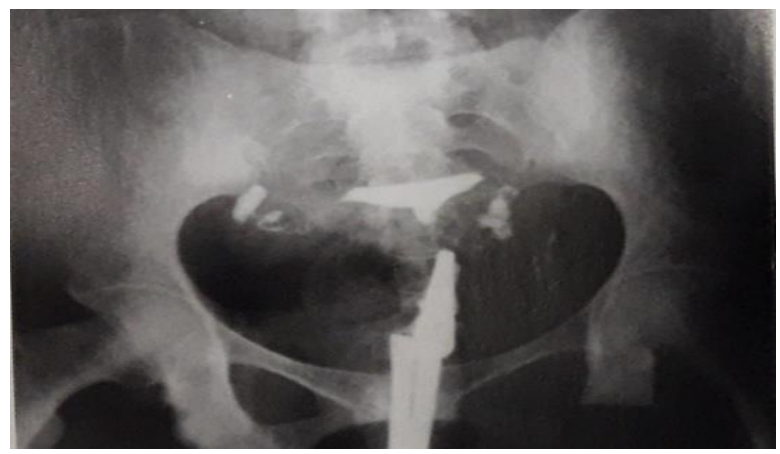

Figure 5: Bilateral hydrosalpinx without any spillage of dye on HSG.

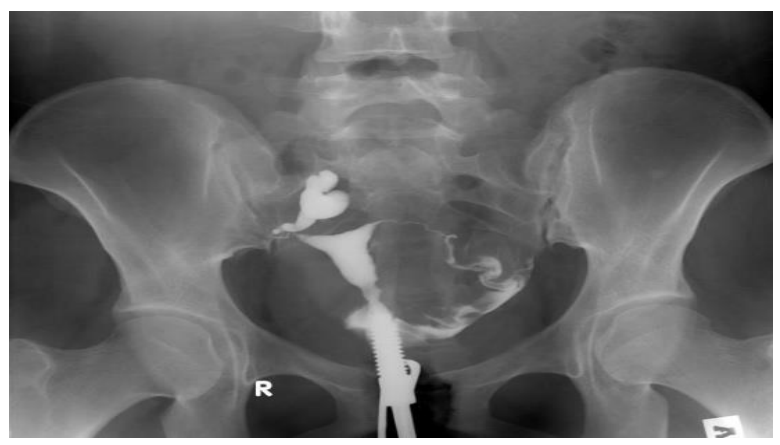

Figure 6: A right sided distal tubal blockage with hydrosalpinx on HSG. 


\section{DISCUSSION}

Infertility is constantly growing in developing countries like India not all patients can afford multiple visits, hence it was needed to set a management protocol in which maximum abnormalities and pathologies can be selected in a single visit. Baseline ultrasonography is needed to be done in all infertile patients to look for antral follicular count, ovaries, pathologies in the adnexa or in the uterus like fibroid, polyp, ovarian mass and also to check for tubal patency. SIS is the single most investigation which when done on day 7-12 can reveal maximum information in an average time span of $10-15$ mins.

In a meta-analysis study by Seshadri et al, SIS was declared to have high degree of diagnostic accuracy in the detection of all types of intrauterine abnormalities with a sensitivity and specificity of 88 and $94 \%$ respectively. ${ }^{7}$ Similarly, Pujar et al, showed slightly better results of SIS in assessing tubal patency and detecting pelvic pathology. ${ }^{8}$ Allahabadia GN et al found $82 \%$ ( 41 out of 50 cases) tubes bilaterally patent by sonosalpingography, $74 \% \quad(37 \quad$ cases $)$ by hysterosalpingography and $82 \%$ (41 cases) by laparoscopy. ${ }^{8}$ Mitri FF et al, who found bilateral patency by sonosalpingography in $31.25 \%$ ( 5 cases out of 16 cases) and by hysterosalpingography in $18.75 \%$ (3 cases). ${ }^{9}$ In the current study bilateral tubal blockage was demonstrated in 4 patients $(8.51 \%)$ by SIS, in 6 patients $(12.7 \%)$ by HSG. Bilateral tubal blockage was reported by Allahabadia GN in $12 \%, 20 \%$ and $12 \%$ cases bt SIS, HSG and diagnostic laparoscopy respectively. ${ }^{10}$ Study done by Beenamol et al, demonstrated bilateral tubal block in $16 \%, 20.8 \%$ and $16 \%$ of cases in SIS, HSG and diagnostic laparoscopy respectively. ${ }^{11}$

The present study showed that SIS is superior to HSG in the evaluation of uterine and tubal factors of female infertility in terms of sensitivity, positive predictive value, negative predictive value and accuracy. Hence, any patient suspected of having an endometrial pathology on conventional TVS scan, SIS must be done as a routine practice.

\section{CONCLUSION}

In a low resource country like India, Saline infusion sonohysterography can prove to be an invaluable diagnostic procedure in meeting the demands of the need for simple, cost-effective, better compliance for the initial work up of an infertile patient on an ambulatory basis and should be incorporated in the initial work up of an infertile woman. HSG and laparoscopic chromopertubation should only be reserved for those with doubtful diagnosis in saline infusion or with long unexplained infertility.
Funding: No funding sources

Conflict of interest: None declared

Ethical approval: The study was approved by the Institutional Ethics Committee

\section{REFERENCES}

1. Dutta DC. Text-book of Gynecology. $7^{\text {th }}$ ed. Infertility. Jaypee Brothers; 2016:186-209.

2. Gaucherand P, Pizcenza JS, Salle B, Rudrigos RC. 'Sonohysterography: Preliminary Investigation. JCU. 1992;28:339-48.

3. Despande P, Deshpande S. Sonohysterosalpingography (SHG): an adjuvant in infertility evaluation'. J Evol Med Dent Sci. 2015;4(10):1628-33.

4. Singh V, Mishra B, Sinha S. Role of saline infusion sonohysterography in infertility evaluation. J Hum Reprod Sci. 2018;11:236-41.

5. Brown SE, Coddington CC, Schnorr J, Toner JP, Gibbons W, Oehninger S. Evaluation of outpatient hysteroscopy, saline infusion hysterosonography, and hysterosalpingography in infertile women: a prospective, randomized study. Fert Ster. 2000;74(5):1029-34.

6. Onwutchekwa CR, Oriji VK. Hysterosalpingographic pattern of infertility in women of reproductive age. J Hum Reprod Sci. 2017;10:178-84.

7. Seshadri S, El Toukhy T, Douiri A, Jayaprakasan K, Khalaf Y. Diagnostic accuracy of saline infusion sonography in the evaluation of uterine cavity abnormalities prior to assisted reproductive techniques: a systematic review and meta analyses. Hum Reprod Update. 2015;21:262-7.

8. Pujar Y, Sherigar B, Patted S, Desai BR, Dhumale H. Comparative evaluation of saline infusion sonohysterography and hysterolaparoscopy for diagnosis of uterine cavity abnormalities and tubal patency in infertility: a one-year cross sectional study. South Asian Fed Obstet Gynecol. 2010;2:133 5.

9. Mitri FF, Mitri FF, Andronikou AD, Perpinyal S, Hofmeyr GJ, Sonnendecker EW. A clinical comparison of sonographic hydrotubation and hysterosalpingography. British J Obst Gynae. 1991;98:1031-6.

10. Allahbadia GN, Nalawade YV, Patkar VD, Niyogi GM, Shah PK. The Sion test. Aust N Z J Obstet Gynaecol. 1992;32:67-70.

11. Beenamol MP, Beenamol S. A comparative study between sonohysterosalpingography and hysterosalpingography in the evaluation of infertility. $\mathbf{J}$ Evol Med Dent Sci. 2013;7:702.

Cite this article as: Bhattacharya R, Ramesh AC. A comparative study of saline infusion sonohysterography and hysterosalpingography for evaluation of female infertility. Int J Reprod Contracept Obstet Gynecol 2020;9:179-83. 